\title{
THE X-RAY LUMINOSITY FUNCTION OF NEARBY RICH AND POOR CLUSTERS OF GALAXIES: A COSMOLOGICAL PROBE
}

\author{
Michael J. Ledlow, ${ }^{1}$ Chris Loken, ${ }^{2}$ Jack O. Burns, ${ }^{2}$ Frazer N. Owen, ${ }^{3}$ and Wolfgang Voges ${ }^{4}$ \\ Received 1998 July 31; accepted 1999 March 8; published 1999 March 16
}

\begin{abstract}
In this Letter, we present a new determination of the local $(z \leq 0.09)$ X-ray luminosity function (XLF) using a large, statistical sample of 294 Abell clusters and the ROSAT All-Sky Survey. Despite the optical selection of this catalog, we find excellent agreement with other recent determinations of the local XLF. Given our large sample size, we have reduced errors by nearly a factor of 2 for $L_{\mathrm{X}(0.5-2.0 \mathrm{keV})} \geq 10^{43} h_{50}^{-2} \mathrm{ergs} \mathrm{s}^{-1}$. We combine our data with previous work to produce the most tightly constrained local determination of the XLF (over 3 orders of magnitude in $L_{\mathrm{X}}$ ) in order to explore possible constraints imposed by the shape of the XLF on cosmological models. A set of currently viable cosmologies is used to construct theoretical XLFs assuming $L \propto M^{p}$ and a $\sigma_{8}-\Omega_{0}$ constraint based on the local X-ray temperature function. We fit these models to our observed XLF and verify that the simplest adiabatic, analytic scaling relation disagrees strongly with observations. If we assume that clusters can be described by the preheated, constant core entropy models of Evrard \& Henry, then the observed XLF is consistent only with $0.1<\Omega_{0}<0.4$ if the energy per unit mass in galaxies is roughly equal to the gas energy (i.e., if $\beta \sim 1$ ).
\end{abstract}

Subject headings: cosmology: observations — galaxies: clusters: general — X-rays: general

\section{INTRODUCTION AND BACKGROUND}

Much of the work on the luminosity distributions of rich clusters has been motivated by the results of Henry et al. (1992), who found evidence for statistically significant negative evolution in the X-ray luminosity function (XLF) (i.e., fewer high$L_{\mathrm{X}}$ clusters at higher $z$ ) at $z \geq 0.3$ for $L_{\mathrm{X}(0.3-3.5 \mathrm{keV})} \geq 5 \times$ $10^{44} h_{50}^{-2} \mathrm{ergs} \mathrm{s}^{-1}$ from 67 clusters in the Einstein Extended Medium-Sensitivity Survey (EMSS). Recently, Vikhlinin et al. (1998) have confirmed the EMSS result at $z>0.3$ for $L_{\mathrm{X}(0.5-2 \mathrm{keV})}>3 \times 10^{44} h_{50}^{-2} \mathrm{ergs} \mathrm{s}^{-1}$ from a $160 \mathrm{deg}^{2}$ survey from pointed ROSAT fields. They found a factor of 3-4 decrease in the number density of these high- $L_{\mathrm{X}}$ clusters as compared to a zero-evolution model. Several other studies have claimed no evolution in the XLF out to redshifts as high as $z=0.8$ (Burke et al. 1997; Jones et al. 1998; Rosati et al. 1998). However, none of these studies have sufficiently large search volumes to address evolution in the XLF at the highest X-ray luminosities and thus do not contradict the original EMSS result.

Of prime importance in any evolutionary study is an accurate determination of the local XLF as a baseline to compare with the distant cluster XLF. Until recently, even the local XLF was quite poorly constrained because of low cluster numbers. The largest local samples compiled to date are the X-ray Brightest Abell Clusters (XBACS; Ebeling et al. 1993, 1996) and the Brightest Cluster Sample (BCS) of Ebeling et al. (1997, 1998). The BCS includes $199 \mathrm{X}$-ray selected clusters down to $\approx 5 \times 10^{42} \mathrm{ergs} \mathrm{s}^{-1}$ in the $0.1-2.4 \mathrm{keV}$ band out to $z \leq 0.3$. Consistent with most previous claims, no evidence was found

\footnotetext{
${ }^{1}$ Institute for Astrophysics, University of New Mexico, Albuquerque, NM 87131; mledlow@wombat.phys.unm.edu.

${ }^{2}$ Office of Research and Department of Physics and Astronomy, University of Missouri Columbia, MO 65211; cloken@hades.physics.missouri.edu, burnsj@missouri.edu.

${ }^{3}$ National Radio Astronomy Observatory, Socorro, NM 87801; fowen@ pilabo.aoc.nrao.edu. The National Radio Astronomy Observatory is operated by Associated Universities, Inc., under contract with the National Science Foundation.

${ }^{4}$ Max-Planck-Institut für Extraterrestrische Physik, Postfach 1603, D-85740, Garching bei München, Germany; whv@mpe.dnet.nasa.gov.
}

for evolution in the XLF within $z \leq 0.2-0.3$ (Ebeling et al. 1998).

We have examined a statistically complete sample of 294 Abell rich clusters within $z \leq 0.09$ using the ROSAT All-Sky Survey (RASS) over the energy band $0.5-2 \mathrm{keV}$ as part of a multiwavelength study of nearby galaxy clusters. Unlike most other studies, our sample is purely optically selected within the criteria for inclusion in Abell's catalog. There is some overlap with both the BCS and XBACS samples, with the primary differences that we have used only Abell's northern catalog (Abell 1958) and our X-ray flux limit is approximately a factor of 8 lower than the BCS sample. Our sample is larger than the BCS, while our volume is nearly 30 times smaller. Given our large sample size, we have reduced statistical errors in the local XLF for $L_{\mathrm{X}} \geq 10^{43} h_{50}^{-2} \mathrm{ergs} \mathrm{s}^{-1}$ by up to a factor of 2 compared to previous work. Combined with the poor cluster XLF of Burns et al. (1996, hereafter BLL96), we examine the composite local XLF over more than 3 orders of magnitude in $L_{\mathrm{X}}$ in order to understand the cosmological constraints imposed by the tight power-law shape noted in BLL96.

In $\S 2$ we describe the sample and the derivation of the local XLF and discuss the limitations imposed by our sample selection. In $\S 3$ we compare our new XLF with previous work. In $\S 4$ we explore the consequences of the shape of the local XLF with regard to Press-Schechter analytic predictions of the mass function and possible constraints on $\Omega_{0}$ and $\Lambda$. We list our conclusions in $\S 5$. We adopt $H_{0}=50 h_{50} \mathrm{~km} \mathrm{~s}^{-1} \mathrm{Mpc}^{-1}$ and $q_{0}=0.5$ when dealing with the observational data.

\section{THE SAMPLE AND DERIVATION OF THE X-RAY LUMINOSITY FUNCTION}

Our cluster sample is derived from Abell's Northern catalog and includes all Abell clusters in the range $0.016 \leq z \leq 0.09$ with galactic absorption less than $0.1 \mathrm{mag}$ at $R$ band $\left(\log N_{\mathrm{H}} \approx 20.73\right)$. See Voges et al. (1999) and Ledlow \& Owen (1995) for more details on the sample selection. The total sample includes 294 Abell clusters. All clusters have measured redshifts, and we include all richness classes in the sample. We calculate a survey area of $14,155 \mathrm{deg}^{2}$ or $34 \%$ of the sky. Within 
our observed volume, we find the number density of clusters to be constant as a function of richness class and redshift, suggesting that our sample is nearly complete and volume limited within the limits of Abell's selection criteria. These findings are consistent with those of Briel \& Henry (1993) and Mazure et al. (1996) with regard to the completeness of Abell's catalog over this redshift regime.

The X-ray luminosity function was derived from images produced by the RASS as described in Voges et al. (1999). Xray luminosities were calculated within a metric aperture of $0.75 h_{50}^{-1} \mathrm{Mpc}$ in diameter over the energy band $0.5-2 \mathrm{keV}$ assuming a thermal spectrum with $T=5 \mathrm{keV}$. Corrections for missing flux were made according to the prescription of Briel $\&$ Henry (1993) (using $\beta=2 / 3$ ) to produce a total $L_{\mathrm{X}}$ for each cluster over our ROSAT band. The primary effect of using a different $\beta$ would be to shift the total luminosities to higher or lower values (a larger $\beta$ results in a smaller correction, thus lower total $L_{\mathrm{X}}$ ), while not significantly changing the shape or amplitude of the XLF within the error bars.

Voges et al. found a total detection rate of $83 \%$ for this sample of Abell clusters. For nondetections, we adopt the $3 \sigma$ upper limits given in their Table 1 . Because of variations in exposure time (and slight variations in galactic absorption) across the sky with the RASS, each cluster has a different flux limit, or maximum volume to which the cluster could have been detected. We follow the prescription of Avni \& Bahcall (1980) and calculate the observed volume separately for each cluster. The volume is evaluated from $z_{\min }=0.016$ to the maximum redshift at which the cluster could have been detected with a $3 \sigma$ confidence. For clusters with only upper limits to $L_{\mathrm{X}}$, we set $z_{\max }$ equal to the redshift of the cluster. The XLF is then found by calculating $d n(L) / d L$ as the sum over all clusters divided by the maximum search volumes of each cluster. Each binned data point is then found by dividing the above sum by the bin width $\left(\Delta L_{\mathrm{X}}\right)$. For the entire sample, we find $\left\langle V / V_{\max }\right\rangle=0.56 \pm 0.02$. Error bars on the data points were calculated assuming Poisson statistics following the prescription of Rosati et al. (1998).

\section{THE X-RAY LUMINOSITY FUNCTION}

In Figure 1, we show the differential XLF for our lowredshift cluster sample. Also on this plot are the measurements of BLL96 derived from 49 poor clusters and the BCS sample of Ebeling et al. (1998). The steady decline in volume density observed in our rich cluster sample for $L_{\mathrm{X}}<10^{43} h_{50}^{-2}$ can be understood from the limitations of Abell's optical selection criteria. Because $L_{\mathrm{X}}$ varies considerably for a given optical richness (Voges et al. 1999), there are a significant number of optically poor clusters with $L_{\mathrm{X}}$ in the range of richness class 0 clusters that are not in our sample. Thus, our sample is truly volume limited only for clusters above this cutoff in $L_{\mathrm{X}}$. Note, however, that for $L_{\mathrm{X}}>10^{43} \mathrm{ergs} \mathrm{s}^{-1}$, our Abell cluster sample and the BCS sample are in excellent agreement. The BCS also extends to higher $L_{\mathrm{X}}$ because of the larger search volume $(z \leq 0.3)$. Our XLF shown in Figure 1 is also consistent with that of Edge et al. (1990) and Briel \& Henry (1993).

The local, differential XLF is remarkably well represented by a power law over more than 3 orders of magnitude in $L_{\mathrm{X}}$. The high-luminosity break in the XLF occurs at greater than $10^{45} h_{50}^{-2} \mathrm{ergs} \mathrm{s}^{-1}$ and can be seen when we include the highest luminosity point from the BCS sample. Using the combined XLF of BLL96 and our new determination of the local richcluster XLF (for $L_{\mathrm{X}}>10^{43} h_{50}^{-2} \operatorname{ergs~\mathrm {s}^{-1}}$ ), we find a power-law

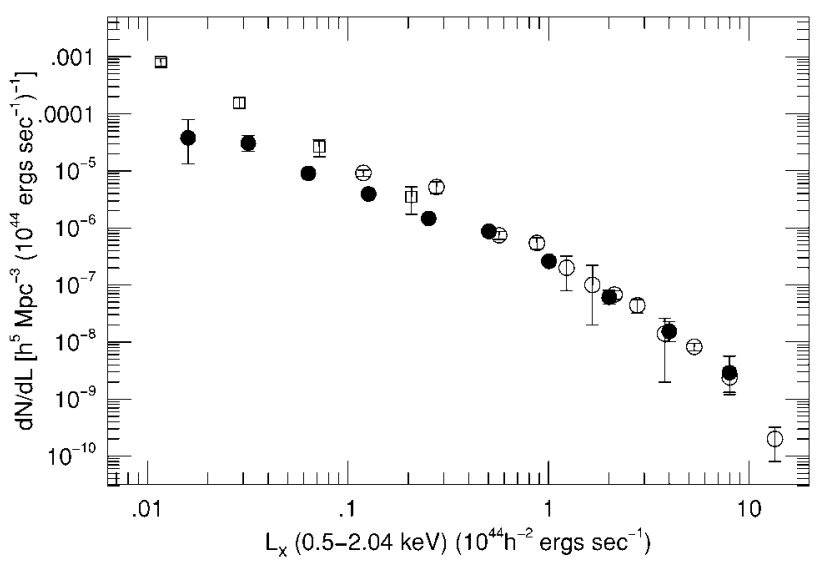

FIG. 1.-X-ray luminosity function derived from our low-redshift $(z \leq$ 0.09) Abell cluster sample (filled circles), the poor-cluster data points from Burns et al. (1996) (open squares), and the XLF from the BCS of Ebeling et al. (1998) (open circles).

fit of the form $\phi(L)=K L_{44}^{-\alpha}$, where $L_{44}$ is the X-ray luminosity in units of $10^{44} \mathrm{ergs} \mathrm{s}^{-1}$ and $K$ is in units of $10^{-7} \mathrm{Mpc}^{-3}$ $L_{44}^{\alpha-1}$. We find best-fit values of $\alpha=1.83 \pm 0.04$ and $K=$ $2.35 \pm_{0.22}^{0.24}$. For completeness, we also fit a Schechter function after including the highest- $L_{\mathrm{X}}$ point from the BCS. For a fit of the form $d N / d L_{\mathrm{X}}=A \exp \left(-L_{\mathrm{X}} / L_{\mathrm{X}}^{*}\right) L_{\mathrm{X}}^{-\alpha}$, we find $A=$ $(2.93 \pm 0.14) \times 10^{-7}\left(\mathrm{Mpc}^{-3} L_{44}^{\alpha-1}\right), \quad L_{\mathrm{X}(0.5-2 \mathrm{keV})}^{*}=5.49 \pm$ $0.39\left(10^{44} \mathrm{ergs} \mathrm{s}^{-1}\right)$, and $\alpha=1.77 \pm 0.01$. These values are consistent within the errors to the BCS, the ROSAT Deep Cluster Survey XLF (Rosati et al. 1998) out to $z=0.6$, and the southern Serendipitous High-Redshift Archival ROSAT Cluster survey (Burke et al. 1997) for $0.3<z<0.7$. Note that these results do not conflict with the claimed negative evolution in the XLF observed by Henry et al. (1992) and most recently by Vikhlinin et al. (1998) at the highest luminosities.

As noted by BLL96, the remarkable power-law shape over such a large range in $L_{\mathrm{X}}$ suggests a continuity in that the bulk $\mathrm{X}$-ray properties of poor clusters must not be fundamentally different from richer systems. We explore the consequences of this result in the next section.

\section{DERIVATION OF THE THEORETICAL X-RAY LUMINOSITY FUNCTION}

In order to assess the constraints our local XLF imposes on cosmological models, we compare it with various analytic predictions. We proceed by using the Press-Schechter (P-S) formalism (e.g., Press \& Schechter 1974; Bond et al. 1991) to construct theoretical mass functions and then convert these to XLFs assuming a form for the X-ray mass-to-light ratio (see Evrard \& Henry 1991, hereafter EH91).

We begin with the set of cosmological models whose parameters are listed in Table 1 . These models form a representative sample of current views since they include open and flat universes spanning a range in $\Omega_{0}$. For each model, the rms density fluctuation on $8 \mathrm{~h}^{-1} \mathrm{Mpc}$ scales $\left(\sigma_{8}\right)$ was determined from the $\sigma_{8}-\Omega_{0}$ relation of Viana \& Liddle (1996) which, in turn, was fixed by the local number density of $7 \mathrm{keV}$ clusters. The Hubble constant was chosen to give an age for the universe of roughly $12.5 \mathrm{Gyr}$ (consistent with globular cluster age determinations; e.g., Chaboyer et al. 1998). For each model we list the relative contributions of matter $\left(\Omega_{0}\right)$, baryonic matter $\left(\Omega_{b}\right)$, and the cosmological constant $\left(\Omega_{\Lambda}\right)$ to the overall energy 
TABLE 1

Cosmological Model Parameters

\begin{tabular}{|c|c|c|c|c|c|c|c|c|}
\hline Model & $\mathrm{ge}^{\mathrm{a}}$ & $H_{0}{ }^{\mathrm{b}}$ & $\Omega_{0}$ & $\Omega_{b}$ & $\Omega_{\Lambda}$ & $\sigma_{8}$ & $p$ & $\beta$ \\
\hline$C D M 4$ & & 70 & 0 & 926 & 0.0 & 467 & & \\
\hline & & 65 & & & & & & \\
\hline & & 65 & & & & & & 14 \\
\hline & & 6 & & & & & & 1.47 \\
\hline & & 60 & 0 & & 0. & 0 & 3.21 & 1.90 \\
\hline & 1 & 60 & & & 0. & & 29 & $40^{+}$ \\
\hline & & & & & & & & 84 \\
\hline & & 8 & & 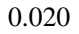 & 0 & & 21 & 1.17 \\
\hline$\Lambda \mathrm{Cl}$ & 12 & 70 & 0.4 & 0.026 & 0.6 & 0.901 & & 1.62 \\
\hline & & 65 & 0.5 & 0.0 & 0.5 & 0.8 & $2.8 \mathrm{c}$ & 1.90 \\
\hline$\Lambda$ CDM5 & 12.2 & 60 & 0.7 & 0.035 & 0.3 & 0.719 & $3.18_{-0.27}^{+0.31}$ & $3.5_{-1}^{+2}$ \\
\hline
\end{tabular}

${ }^{\mathrm{a}}$ Current age of universe in Gyr.

${ }^{\mathrm{b}}$ Hubble constant in units of $\mathrm{km} \mathrm{s}^{-1} \mathrm{Mpc}^{-1}$.

density. Power spectra for all of the models were generated using the code described in Klypin \& Holtzman (1997), and then P-S mass functions (with $\delta_{c}=1.3$ ) were computed at $z=0$.

Our P-S mass functions can be converted to XLFs by assuming a form for the mass-luminosity relation and correcting to our bandpass. We assume that the bolometric X-ray luminosity is related to cluster mass as $L_{\text {bol }}=c M^{p}$ and will later fit for the parameters $c$ and $p$. There exist at least two theoretical predictions for the value of the exponent $p$. The self-similar model of Kaiser (1986), derived assuming a power-law initial perturbation spectrum and purely adiabatic gas physics, predicts $p=4 / 3$, but it is well known that this fails to give the correct shape for the XLF (e.g., EH91; see also below). However, preheating of the intracluster medium at an early epoch (possibly by galaxy formation) results in a different scaling relation and also resolves several discrepancies between theoretical and observational results concerning evolution in the XLF (e.g.,

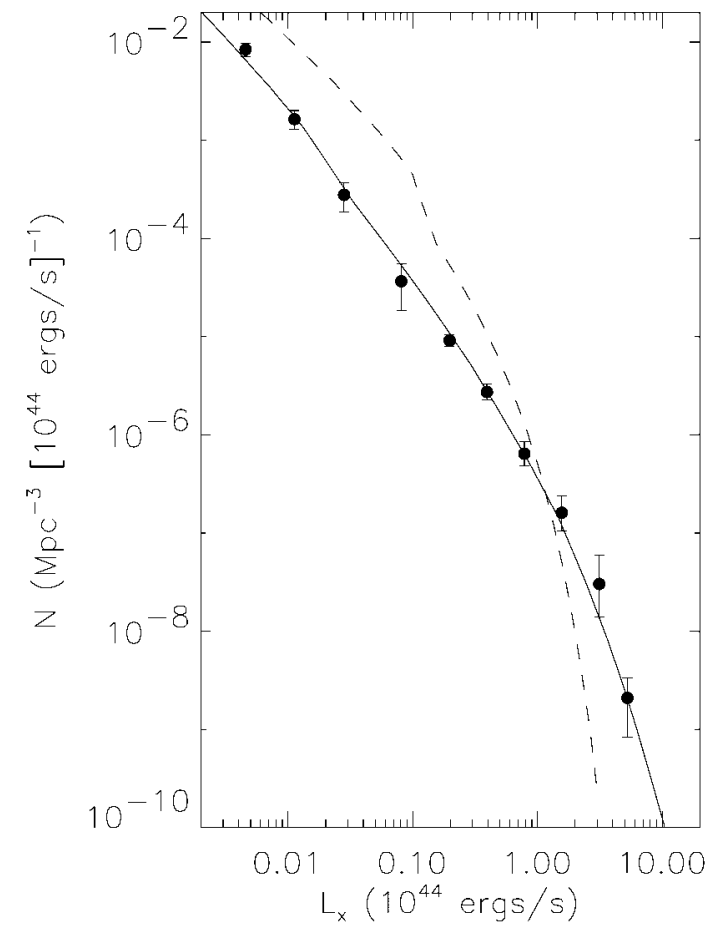

Evrard 1990; Navarro, Frenk, \& White 1995). For the case of a constant entropy core, EH91 derived a scaling that implies $p=(10 \beta-3) / 3 \beta$, where $\beta=\mu m_{p} \sigma^{2} / k T$ is the usual ratio of dark matter to gas "temperatures."

We correct our bolometric luminosities to the $0.5-2 \mathrm{keV}$ bandpass by calculating temperatures and applying a correction appropriate for a plasma with a metallicity of $Z=0.3 Z_{\odot}$. Specifically, the temperature corresponding to a given mass can be calculated from the analytic $M-T$ relation derived from the virial theorem (e.g., Bryan \& Norman 1998): $k T=$ $(1.39 / \beta)\left(M / 10^{15} M_{\odot}\right)^{2 / 3} \Delta_{c}^{1 / 3} h^{2 / 3} \mathrm{keV}$, where $\Delta_{c}$ is the current density contrast within the cluster virial radius. The luminosity in our bandpass is then calculated by applying the usual bremmstrahlung correction factor as well as a multiplicative factor to account for the presence of metals (Bryan \& Norman 1998, eq. [21]).

Using the relation for $L_{\mathrm{bol}}$ and the bandpass correction, we converted our P-S mass functions to differential luminosity functions and made $\chi^{2}$ fits to a subset of the observational data. The observational points used in the fits are all four poor cluster points (BLL96), the five highest luminosity Abell cluster points, and the highest luminosity BCS point from Figure 1. We first set $\beta=1$ in the $M-T$ relation and fit for $c$ and $p$. The fitted value for $p$ is included in Table 1, and examples of two of the fits are shown in Figure 2. The dashed curve in Figure $2 a$ is the best fit when the exponent is kept fixed at the analytic prediction $p=4 / 3$. Clearly, the shape of the XLF derived using this prediction is in gross disagreement with the observed function. Figure $2 b$ also shows the importance of the low- and/or high-luminosity data points. If only our five Abell cluster data points are used (dotted line), the fitted value of $p$ increases by at least 0.2 in all cases (from $p=3.18$ to $p=3.88$ in this case). We get virtually identical results if we redo our fits

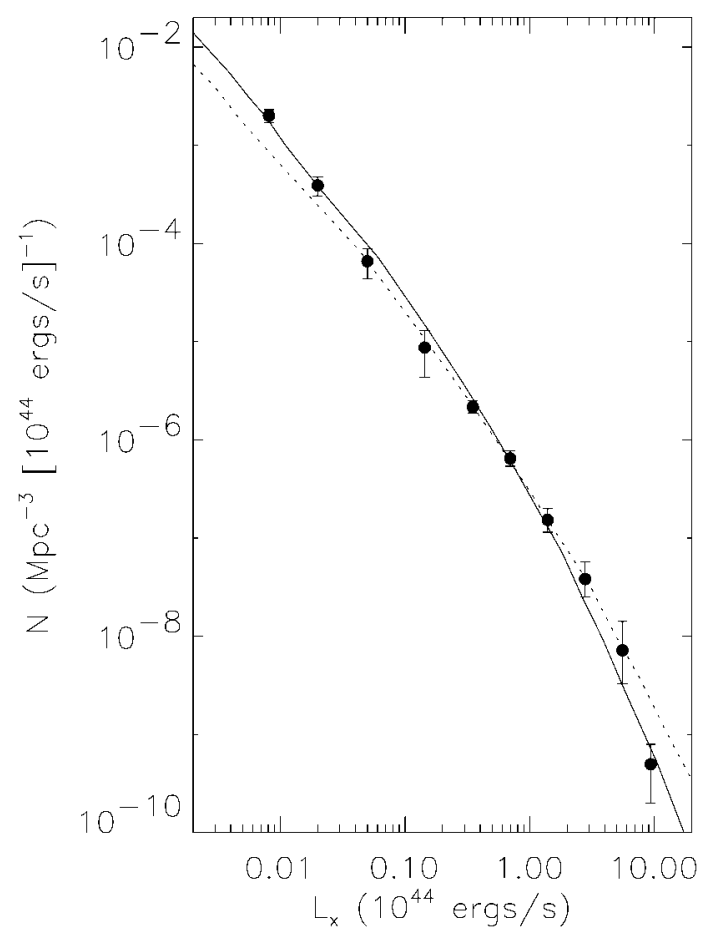

FIG. 2.-Fits to the observed XLF for two models. The solid lines are the best fits to a subset of the observational XLF points for models $\Lambda$ CDM1 (left) and $\Lambda$ CDM5 (right). The dashed line in the left panel is the best fit to the models. The dotted line in the right panel represents the best fit to the data when the BCS and poor cluster points are ignored. 
without the BCS point, whereas dropping the poor cluster points results in slightly greater discrepancies.

If we invoke the constant entropy core model of EH91, then the exponent in the mass-luminosity relation is actually a function of $\beta[p=(10 \beta-3) / 3 \beta]$. In this case, we fit for $c$ and $\beta$ and find the values listed in Table 1 . Interestingly, only the models with $0.1<\Omega_{0}<0.4$ are consistent with the expected value $\beta \sim 1$. (A recent observational analysis found $\beta=$ $0.94 \pm 0.08$ [Lubin \& Bahcall 1993], which is in good agreement with numerical results [e.g., Eke, Navarro, \& Frenk 1998].) Thus, if the constant entropy core model of EH91 applies, the present-day XLF observations suggest a low-density universe but cannot distinguish between open and flat cases.

\section{CONCLUSIONS}

Starting from an optically selected, statistical sample of Abell clusters, we have made a new determination of the local XLF to compare to previous work and more distant cluster samples. Our cluster sample is larger than all previous studies and is contained within a smaller volume. For this reason, we have reduced statistical uncertainties in the local XLF by nearly a factor of 2 for a limited range in $L_{\mathrm{X}}\left(L_{\mathrm{X}}>10^{43} h_{50}^{-2} \mathrm{ergs} \mathrm{s}^{-1}\right)$. It is only for $L_{\mathrm{X}}<10^{43} \mathrm{ergs} \mathrm{s}^{-1}$ that incompleteness due to the optical selection of our sample is apparent. The observed incompleteness is not a failing in Abell's catalog, but rather results from the contribution of poor clusters and groups below Abell's richness limit.

Combined with the poor-cluster XLF of BLL96, we have examined the local XLF over nearly 3 orders of magnitude in $L_{\mathrm{X}}$. We find that the local XLF is remarkably well represented by a power law over nearly this entire range in $L_{\mathrm{X}}$. This is significant evidence that hierarchical formation results in sim- ilar cluster properties over a large range in $L_{\mathrm{X}}$ and mass. Including the brightest $L_{\mathrm{X}}$ clusters from the BCS sample that fall above the break in the XLF at $L_{\mathrm{X}}>10^{45} h_{50}^{-2}$ ergs s${ }^{-1}$, we also performed a Schechter-function fit which is in good agreement with other recent surveys to much higher redshift $(z<0.7)$, confirming a lack of significant evolution at these luminosities.

We have used our new local XLF to derive a constraint on $\Omega_{0}$. This would appear to contradict a common claim that the $\sigma_{8}-\Omega_{0}$ degeneracy can be broken only by including the evolution with redshift (e.g., Bahcall \& Fan 1998). In fact, P-S mass functions for combinations of $\sigma_{8}$ and $\Omega_{0}$ that satisfy a $\sigma_{8}-\Omega_{0}$ constraint differ in shape. Borgani et al. (1999) have recently used the shape of the local XLF in order to constrain $\sigma_{8}-\Omega_{0}$ and the shape of the $L-T$ relation. Including clusters at higher redshift, they concluded that $\Omega_{0}=0.4_{-0.2}^{+0.3}$ for open models and $\Omega_{0} \leq 0.6$ for flat models assuming no evolution in the $L-T$ relation, both of which are consistent with our results. In this work, we have used the shape of the local XLF, the local number density of $7 \mathrm{keV}$ clusters, and the P-S formalism in order to constrain the cluster $M-L$ relation: $L_{\mathrm{X}} \propto M^{p}$. There is a clear trend for $p$ to increase with $\Omega_{0}$ (see also Mathiesen \& Evrard 1998). None of the theoretical models are consistent with the analytic prediction $p=4 / 3$ from Kaiser (1986). If we adopt the constant core entropy model of EH91 and the additional constraint that $\beta \approx 1$, the shape of the local XLF suggests that $0.1 \leq \Omega_{0} \leq 0.4$, with no constraint on $\Lambda$.

This work was supported in part by NASA grants NAG56739 and NAGW-3152 and NSF grant AST-9896039. We thank Anatoly Klypin and Jon Holtzman for use of their code and for useful discussions. We also thank Neta Bahcall and an anonymous referee for helpful suggestions. J. O. B. and F. N. O. thank MPE for their hospitality during several visits.

\section{REFERENCES}

Abell, G. O. 1958, ApJS, 31, 211

Avni, Y., \& Bahcall, J. N. 1980, ApJ, 235, 694

Bahcall, N. A., \& Fan, X. 1998, ApJ, 504, 1

Bond, J. R., Cole, S., Efstathiou, G., \& Kaiser, N. 1991, ApJ, 379, 440

Borgani, S., Rosati, P., Tozzi, P., \& Norman, C. 1999, ApJ, in press (astro$\mathrm{ph} / 9901017)$

Briel, U. G., \& Henry, J. P. 1993, A\&A, 278, 379

Bryan, G., \& Norman, M. L. 1998, ApJ, 495, 80

Burke, D. J., Collins, C. A., Sharples, R. M., Romer, A. K., Holden, B. P., \& Nichol, R. C. 1997, ApJ, 488, L83

Burns, J. O., Ledlow, M. J., Loken, C., Klypin, A., Voges, W., Bryan, G. L., Norman, M. L., \& White, R. A. 1996, ApJ, 467, L49 (BLL96)

Chaboyer, B., Demarque, P., Kernan, P. J., \& Krauss, L. M. 1998, ApJ, 494, 96

Ebeling, H., Edge, A. C., Böhringer, H., Allen, S. W. Crawford, C. S., Fabian, A. C., Voges, W., \& Huchra, J. P. 1998, MNRAS, 301, 881

Ebeling, H., Edge, A. C., Fabian, A. C., Allen, S. W., Crawford, C. S., \& Böhringer, H. 1997, ApJ, 479, L101

Ebeling, H., Voges, W., Böhringer, H., \& Edge, A. C. 1993, A\&A, 275, 360

Ebeling, H., Voges, W., Böhringer, H., Edge, A. C., Huchra, J. P., \& Briel, U. G. 1996, MNRAS, 283, 1103

Edge, A. C., Stewart, G. C., Fabian, A. C., \& Arnaud, K. A. 1990, MNRAS, 245,559
Eke, V. R., Navarro, J. F., \& Frenk, C. S. 1998, ApJ, 503, 569

Evrard, A. E. 1990, ApJ, 363, 349

Evrard, A. E. \& Henry, J. P. 1991, ApJ, 383, 95 (EH91)

Henry, J. P., Gioia, I. M., Maccacaro, T., Morris, S. L., Stocke, J. T., \& Wolter, A. 1992, ApJ, 386, 408

Jones, L. R., Scharf, C., Ebeling, H., Perlman, E., Wegner, G., Malkan, M., \& Horner, D. 1998, ApJ, 495, 100

Kaiser, N. 1986, MNRAS, 219, 785

Klypin, A. \& Holtzman, J. 1997, preprint (astro-ph/9712217)

Ledlow, M. J., \& Owen, F. N. 1995, AJ, 109, 853

Lubin, L. M., \& Bahcall, N. A. 1993, ApJ, 415, L17

Mathiesen, B., \& Evrard, A. E. 1998, MNRAS, 295, 769

Mazure, A., et al. 1996, A\&A, 310, 31

Navarro, J. F., Frenk, C. S., \& White, S. D. M. 1995, MNRAS, 275, 720

Press, W. H., \& Schechter, P. 1974, ApJ, 187, 425

Rosati, P., Ceca, R. D., Norman, C., \& Giacconi, R. 1998, ApJ, 492, L21

Viana, P. T. P., \& Liddle, A. R. 1996, MNRAS, 281, 323

Vikhlinin, A., McNamara, B. R., Forman, W., Jones, C., Quintana, Q., \& Hornstrup, A. 1998, preprint (astro-ph/9803101)

Voges, W., Ledlow, M. J., Burns, J. O., Owen, F. N., \& Smith, C. 1999, AJ, submitted 